University of New Hampshire

University of New Hampshire Scholars' Repository

Physics Scholarship

Physics

$2-26-2013$

\title{
Collapse of the Cooper pair phase coherence length at a superconductor-to-insulator transition
}

Shawna M. Hollen

University of New Hampshire - Main Campus

G. E. Fernandes

Brown University

J. M. Xu

Brown University

J M. Valles Jr.

Brown University

Follow this and additional works at: https://scholars.unh.edu/physics_facpub

Part of the Physics Commons

\section{Recommended Citation}

S. M. Hollen, G. E. Fernandes, J. M. Xu, and J. M. Valles, 'Collapse of the Cooper pair phase coherence length at a superconductor-to-insulator transition', Physical Review B, vol. 87, no. 5, Feb. 2013.

This Article is brought to you for free and open access by the Physics at University of New Hampshire Scholars' Repository. It has been accepted for inclusion in Physics Scholarship by an authorized administrator of University of New Hampshire Scholars' Repository. For more information, please contact Scholarly.Communication@unh.edu. 


\title{
Cooper-pair insulator phase in superconducting amorphous Bi films induced by nanometer-scale thickness variations
}

\author{
S. M. Hollen, ${ }^{1}$ H. Q. Nguyen,,${ }^{1, *}$ E. Rudisaile, ${ }^{1}$ M. D. Stewart Jr., ${ }^{1, \dagger}$ J. Shainline,,${ }^{1, \dagger}$ J. M. Xu,${ }^{1,2,3}$ and J. M. Valles Jr. ${ }^{1}$ \\ ${ }^{1}$ Department of Physics, Brown University, Providence, Rhode Island 02912, USA \\ ${ }^{2}$ Division of Engineering, Brown University, Providence, Rhode Island 02912, USA \\ ${ }^{3}$ WCU program, Seoul National University, Seoul, Republic of Korea, Korea
}

(Received 27 May 2011; published 25 August 2011)

\begin{abstract}
Ultrathin films near the quantum insulator-superconductor transition (IST) can exhibit Cooper-pair transport in their insulating state. This Cooper-pair insulator (CPI) state is achieved in amorphous Bi films evaporated onto substrates with a topography varying on lengths slightly greater than the superconducting coherence length. We present evidence that this topography induces film thickness and corresponding superconducting coupling constant variations that promote Cooper-pair island formation. Analyses of many thickness-tuned ISTs show that weak links between superconducting islands dominate the transport. In particular, the IST occurs when the link resistance approaches the resistance quantum for pairs. These results support conjectures that the CPI is an inhomogeneous state of matter.
\end{abstract}

DOI: 10.1103/PhysRevB.84.064528

PACS number(s): 74.81.-g, 64.70.Tg, 74.62.-c, 74.78.-w

Investigators have focused on determining the elementary constituents of the insulating phase of thin films near the insulator-superconductor transition (IST) for more than 20 years. ${ }^{1}$ In most homogeneously disordered thin films, the insulator is now understood to be composed of unpaired electrons that are weakly localized by disorder. ${ }^{2-6}$ In some composite and highly disordered films, such as In oxide and TiN, a less familiar Cooper-pair insulator (CPI) state obtains that appears to support remnants of superconductivity. ${ }^{7,8}$ Experiments probing their electronic transport have revealed remarkable deviations from conventional behavior. Most significantly, the magnetoresistance of films near the IST forms a giant peak at $H \sim H_{c 2}$. Additionally, the insulators close to the magnetic field-tuned IST often exhibit a reentrant drop in resistance with temperature and simply activated transport at low temperatures. ${ }^{7-9}$ Theoretical descriptions of this CPI phase often presume that disorder or magnetic field creates islands of Cooper pairs embedded in a nonsuperconducting background. ${ }^{10-13}$ Although the description of the CPI as an inhomogeneous phase of matter is consistent with observations, the experimental support is limited. The most direct support provided so far is scanning tunneling microscopy (STM) measurements which show evidence of spatial superconducting gap variations of $\lesssim 20 \%$ in superconducting films of $\mathrm{InO}_{x}$ and TiN. ${ }^{14,15}$ The observed variations become more pronounced as the films near the IST. In addition, experiments showing behavior near the IST to depend on the size of the sample (from submicrometer to millimeter) suggest that inhomogeneities are important to the description of the CPI phase. ${ }^{16,17}$

Recently, it was established that homogeneously disordered $a$-Bi films can be induced to form a CPI state instead of the unpaired insulator state by depositing them on an anodized Al oxide substrate patterned with a nanohoneycomb (NHC) array of holes [see the insets in Figs. 1(a) and 1(b)]. Near the IST, these insulating NHC $a$-Bi films exhibit the characteristic transport signatures of the CPI phase. ${ }^{18,19}$ Moreover, the insulators are confirmed to contain Cooper pairs through Little-Parks-like magnetoresistance oscillations induced by the hole array. ${ }^{18}$ How the NHC substrates induce the formation of the CPI state in these films is the primary focus of this paper.

We report that the NHC films contain thickness and, consequently, superconducting coupling constant variations that form because the holey substrates have a nonuniform topography. These nanometer-scale inhomogeneities produce a network of thicker, superconducting regions connected by weak links of disordered metal. The average weak link conductance assumes an apparently universal value comparable to the quantum conductance for pairs at the IST, suggesting that the weak links control the localization of the Cooper pairs. This paper presents a clear mechanism for driving an amorphous film into an insulating phase without destroying the Cooper pairs.

NHC $a$-Bi thin films are grown by thermally evaporating $\mathrm{Sb}$ and then $\mathrm{Bi}$ onto an anodized aluminum oxide $(\mathrm{AAO})^{20}$ substrate held at $8 \mathrm{~K}$ in a dilution refrigerator cryostat. Reference films are grown simultaneously on a fire-polished glass substrate. Film thicknesses are measured with a quartz crystal microbalance. The semiconducting Sb underlayer $(d \simeq$ $1 \mathrm{~nm}$ ) promotes the surface wetting of the subsequently deposited Bi. Previous STM analyses of quench condensed metal films on semiconductors indicate that the metal atoms stick where they land to form a homogeneous amorphous film. ${ }^{21}$ Thus, the films assume the holey geometry of the underlying AAO substrate [see the insets in Figs. 1(a) and 1(b)]. In the nine different $\mathrm{AAO}$ substrates used in these experiments the average hole radii range from 14 to $28 \pm 3 \mathrm{~nm}$ with an average center-to-center hole spacing of $100 \pm 5 \mathrm{~nm}$ in each substrate. The order in the hole arrangement was intentionally varied using two different anodization processes [see the insets in Figs. 1(a) vs 1(b)]. Sheet resistances as a function of temperature $R(T)$ were measured in situ on $1.5-\mathrm{mm}$-square areas defined by predeposited Ge/Au contacts using four-point ac and dc methods. The geometry of each of the nine hole arrays was characterized using scanning electron microscope images and the surface topography of six of the nine was measured using tapping mode atomic force microscopy (AFM) over a $1 \mu \mathrm{m}^{2}$ area. In representative cases, these measurements were performed on the bare substrate as well as the surface of 


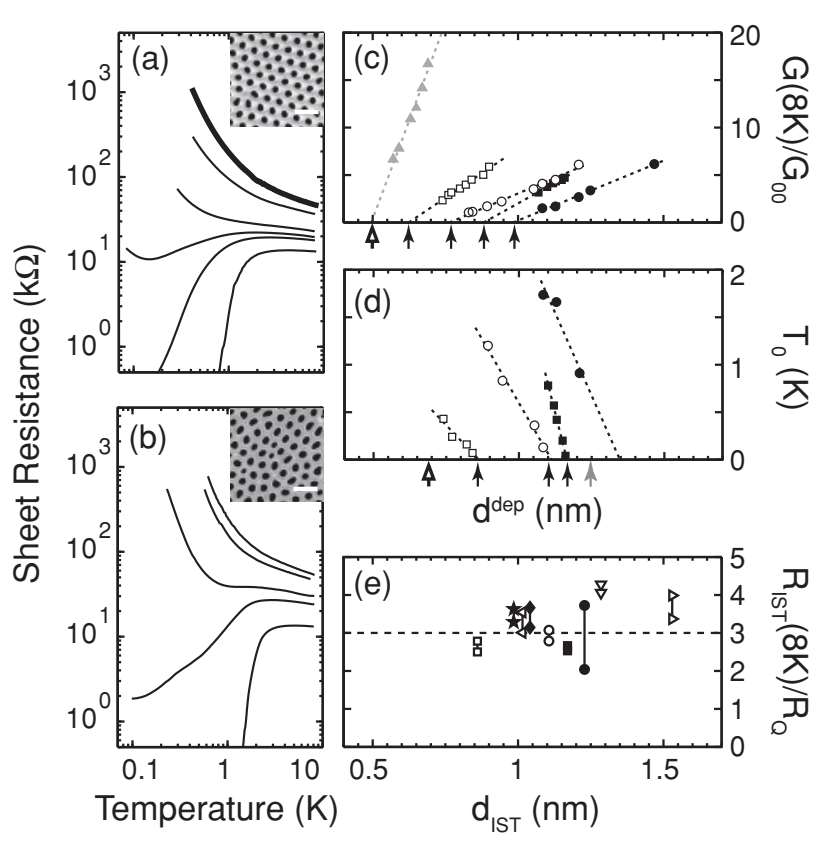

FIG. 1. Characteristics of thin films on nanohoneycomb substrates. (a) and (b) Insulator superconductor transitions tuned by thickness of $a$-Bi on more and less ordered hole arrays. Film thicknesses in (a) from the top down are 0.89, 0.94, 1.05, 1.08, 1.13, and $1.21 \pm 0.1 \mathrm{~nm}$ and in (b) are 1.08, 1.13, 1.21, 1.25, and $1.47 \pm 0.1$ nm. Insets: Postexperiment SEM images. Center-to-center hole spacing: $100 \pm 5 \mathrm{~nm}$. Radii: (a) 27 and (b) $28 \pm 3 \mathrm{~nm}$. The scale bar spans $200 \mathrm{~nm}$. (c) Conductance normalized to the conductance scale for weak localization phenomena $\left[G_{00}=e^{2} /\left(2 \pi^{2} \hbar\right) \approx 1 / 81 \mathrm{k} \Omega\right]$ and (d) activation energies for films on four different NHC substrates. Gray triangles in (c) show data for a reference film. Identical shapes are used when measurements were performed during the same experimental run (on side-by-side NHC substrates). Unfilled and filled symbols represent data from more and less ordered hole arrays, respectively. Arrows on the $x$ axis indicate critical thicknesses for conductivity and SC, and open arrows indicate critical thicknesses in reference films for conductivity, $0.5 \mathrm{~nm}$, and superconductivity, $0.7 \mathrm{~nm}$. The gray arrow in (d) indicates the measured thickness of the first SC film in the filled circle series. This value is a more accurate measure of $d_{\mathrm{IST}}$ than the linear extrapolation. In this case only, we use this value in place of the intercept. Data from the experiment in (a) and (b) are shown as circles. For squares, $r_{\text {hole }}=16$ (unfilled) and 15 (filled) $\pm 3 \mathrm{~nm}$. The dashed lines are least-squares linear fits to the data. (e) Normal-state $(T=8 \mathrm{~K})$ critical sheet resistance for the last insulating and first SC films of the IST of nine different NHC substrates. The horizontal dashed line at $3 R_{\mathrm{Q}}$ is a guide to the eye.

the final quench condensed films $(d<2 \mathrm{~nm})$ postexperiment (ex situ). We observed no significant differences in the measured topography predeposition and postdeposition, as is expected of quench condensed thermal evaporation on a nonuniform surface (detailed in calculations below).

Here we focus on the systematics of thickness tuned ISTs in these films. We pay particular attention to how the critical thicknesses for the onset of conduction $\left(d_{\mathrm{Gc}}\right)$ and superconduction $\left(d_{\text {IST }}\right)$ vary with substrate qualities and also point out the relative invariance of the critical normal-state sheet resistance $\left(R_{\mathrm{IST}}\right)$. The ISTs, shown in Figs. 1(a) and 1 (b), differ significantly from those of their unpatterned, or "reference," counterparts (see Ref. 4 for an example). The $R(T)$ evolve with deposited thickness $d^{\text {dep }}$ from a monotonic rise as $T \rightarrow 0$ that is exponential rather than logarithmic, to develop a reentrant dip not seen in reference films, to finally drop steadily into the SC state. The exponential rise indicates thermally activated transport $R(T) \propto e^{T_{0} / T} \cdot{ }^{18}$ For all the ISTs, the normal-state sheet conductance $G(8 \mathrm{~K})=1 / R(8 \mathrm{~K})$ grows linearly with deposited film thickness $d^{\text {dep}}$, starting at a critical thickness $d_{\mathrm{Gc}}$ [Fig. 1(c)]. This linear behavior reflects homogeneous rather than granular film growth, which would appear as an exponential dependence. ${ }^{22}$ Consistently, the slope $d G(8 \mathrm{~K}) / d d^{\text {dep }}$ is smaller and the intercept $d_{\mathrm{Gc}}$ is larger for the NHC films compared to reference films. $T_{0}$ decreases continuously on approaching the IST (also shown in Refs. 18, 19, and 23). We define the critical thickness for the IST, $d_{\mathrm{IST}}$, as the thickness at which $T_{0}$ linearly extrapolates to zero. Notice that similar to $d_{\mathrm{Gc}}, d_{\mathrm{IST}}$ is larger for $\mathrm{NHC}$ films than reference films. Finally, $R_{\mathrm{IST}}$ shows little variation over nine different substrates that exhibit a wide range of $d_{\text {IST }}$ [see Fig. $1(\mathrm{e})$ ]. The $R_{\mathrm{IST}} \mathrm{S}$ cluster around $R_{\mathrm{IST}} \approx 3 R_{\mathrm{Q}}$, where $R_{\mathrm{Q}}=h /(2 e)^{2}$ is the quantum of resistance for pairs.

Topographical analyses of AAO substrates using AFM images show that the minimum film thickness in critical NHC films (at $d^{\text {dep }}=d_{\mathrm{Gc}}$ and $d_{\mathrm{IST}}$ ) correlates with critical thicknesses in the reference films $d_{\mathrm{Gc}}^{\mathrm{ref}}$ and $d_{\mathrm{IST}}^{\mathrm{ref}}$. As pointed out previously, ${ }^{24}$ the NHC substrate topography $h(x, y)$ shows regular variations around holes, which makes the thermally evaporated film thickness a function of position $d(x, y)$. For a uniform flux of atoms impinging parallel to the average normal to the substrate,

$$
d(x, y)=d^{\mathrm{dep}} \frac{1}{\sqrt{1+(\nabla h)^{2}}},
$$

where $d^{\text {dep }}$ is the thickness measured by a (flat) quartz crystal microbalance. Figure 2(a) shows the topography of the AAO substrate. Six peaks, visible as bright spots, surround each of the holes. A line scan between two neighboring peaks shows a single central minimum in the height [Fig. 2(b)]. Using Eq. (1), we calculate thickness profiles along this link corresponding to the onset of conduction $\left(d^{\text {dep }}=d_{\mathrm{Gc}}\right.$ ), an insulating film $\left[d_{\mathrm{Gc}}<d^{\mathrm{dep}}<d_{\mathrm{IST}}\right.$, bold in Fig. 1(a)], and the onset of SC $\left(d^{\text {dep }}=d_{\text {IST }}\right)$ [see Figs. 2(c)-2(e)]. All show two minima that correspond to the steepest regions of the height profile. We show the superconducting coherence length of a reference film of the same $d^{\text {dep }}, \xi \approx 15 \mathrm{~nm}$, in Fig. 2(c) for comparison to the size of the undulations. $\xi$ is approximated from measurement of the upper critical field in reference films. ${ }^{25}$ At the critical thicknesses $d_{\mathrm{Gc}}$ and $d_{\mathrm{IST}}$ the minima coincide with critical thicknesses in reference films $d_{\mathrm{Gc}}^{\text {ref }}$ (pink line at $0.5 \mathrm{~nm}$ ) and $d_{\mathrm{IST}}^{\mathrm{ref}}$ (blue line at $0.7 \mathrm{~nm}$ ). These observations intimate that NHC films conduct or SC when the thinnest regions, the weak links, reach the appropriate critical thickness. Calculations of the weak link thicknesses averaged over each of the six substrates analyzed $\bar{d}^{\text {links }}$ strongly support this suggestion. Figure 2(f) compares $\bar{d}^{\text {links }}$ at NHC film critical thicknesses with the critical thicknesses for conductivity and SC of reference films. It is evident that for all but one of the substrates $\bar{d}^{\text {links }}$ at critical $d^{\text {dep }}$ corresponds to critical thicknesses in reference films.

It is noteworthy that disorder in the geometry of the hole array appears to exert little influence on the IST. If such 

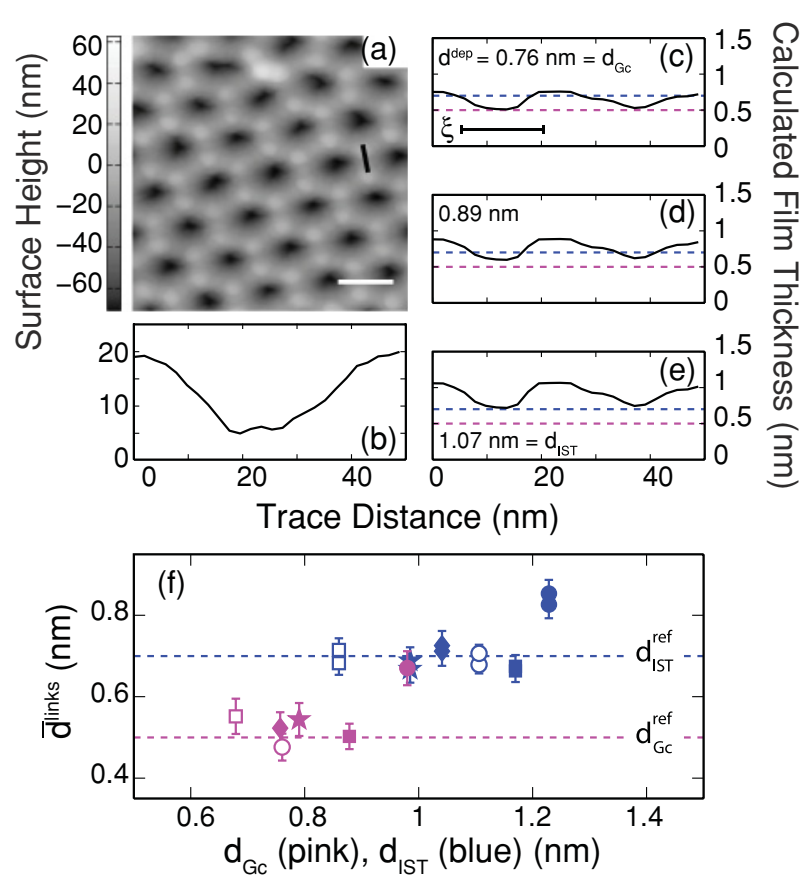

FIG. 2. (Color) NHC film topography. (a) AFM image showing the topography of the substrate in Fig. 1(a). The scale bar (white line) spans $100 \mathrm{~nm}$. (b) Height profile along the trace (black line) in (a). (c)-(e) Film thicknesses along the trace in (a) for $d^{\text {dep }}$ at (c) the onset of conduction, (d) a representative insulating film [bold in Fig. 1(a)], and (e) the IST. Pink and blue lines indicate the critical thicknesses for conductivity and SC, respectively, for a reference film. In (c), the SC coherence length $\xi$ in a reference film of comparable thickness is shown for feature size comparison. (f) Average link thickness for NHC films at their transitions to conductivity $\left(d_{\mathrm{Gc}}\right.$, pink) and SC $\left(d_{\mathrm{IST}}\right.$, blue) for six different substrates. Critical thicknesses for SC are shown by plotting the link thicknesses of the last insulating and first SC films spanning the IST.

geometrical disorder were important, then one would expect $d_{\text {IST }}$ to be consistently lower for the more ordered arrays. The data in Fig. 1(e), however, show that the $d_{\mathrm{IST}}$ 's for the more and less disordered substrates intersperse.

Full two-dimensional maps of the local film thickness $d(x, y)$ reveal a compelling picture: $\mathrm{SC}$ and normal regions coexist in NHC films through the IST (Fig. 3). These maps were generated by applying Eq. (1) to the AFM image in Fig. 2(a) for the series of depositions in Figs. 2(c)-2(e). The color scale indicates regions that are SC, resistive, and insulating according to their behavior at those thicknesses in a reference film. The right-hand axis of the figure further converts $d(x, y)$ to a local $T_{\mathrm{c}}$ map $T_{\mathrm{c}}(x, y)$, using the relationship between $T_{\mathrm{c}}^{\mathrm{ref}}$ and $d^{\text {ref }}$ observed here and in many amorphous film systems: ${ }^{4,26} T_{\mathrm{c}}^{\text {ref }}=T_{\mathrm{c}}^{\text {bulk }}\left(1-d_{\text {IST }}^{\text {ref }} / d^{\text {dep }}\right)$. For $a$-Bi, $T_{\mathrm{c}}^{\text {bulk }}=6 \mathrm{~K}$ and $d_{\mathrm{IST}}^{\mathrm{ref}}=0.7 \mathrm{~nm} .{ }^{27}$ Note that the $T_{\mathrm{c}}$ maps are better thought of as coupling constant maps since they do not include proximity effects. It is evident that SC islands form and grow where the substrate is flattest, such that there are generally 12 islands surrounding each hole, one for each peak and valley. Through the IST, the SC islands are larger than the coherence length in these films $(\xi \approx 15 \mathrm{~nm})$. Each of the islands has a larger $T_{\mathrm{c}}$ at its center than on its edges and resistive film regions surround the islands. With increasing thickness, the intervening resistive regions shrink until the $\mathrm{SC}$ islands coalesce (see the discussion of Fig. 4 below). The $T_{\text {c }}$ contour plot, Fig. 3(d), emphasizes that $T_{\mathrm{c}}$ in the regions between islands at their coalescence is finite, but much less than the maximum at the islands' centers. Thus, these regions likely act as weak links.

The color maps of Fig. 3 suggest a network model for NHC films that provides a clue to the origin of the apparently universal normal-state resistance at the IST. In the model, the $\mathrm{SC}$ islands become highly conductive regions connected by resistive weak links as shown in Fig. 3(e). The resistance of the basic building block of this network, which is equivalent to the macroscopic sheet resistance, is $3 R^{\text {link }}$. Thus, according to Fig. 1(f), at the IST $R_{\mathrm{IST}}^{\text {link }} \approx R_{\mathrm{Q}}$. The observation of a critical normal-state resistance in a network model of NHC films suggests that they are behaving as a resistively shunted Josephson junction array. Theory and experiment on fabricated arrays show that they undergo an insulator-to-superconductor transition when the shunt resistance is comparable to $R_{\mathrm{Q}}$ and the superconducting islands are in the extreme quantum limit, where charging energies are large. ${ }^{28,29}$ The islands evident in the images of the NHC films are small enough to be in the extreme quantum limit. The weak links between the islands probably have the low-energy degrees of freedom necessary to cause dissipation. In addition, both the fabricated arrays and the NHC films exhibit a reentrant dip in $R(T)$ in the insulating films closest to the IST.

The evolution of the distribution of SC island sizes with film deposition provide further support that the weak links control the IST. Figure 4(a) presents histograms of the SC island sizes for the experiment of Fig. 1(a) for five thicknesses. For insulating films with $d^{\text {dep }}<0.89 \mathrm{~nm}$, the island size is relatively uniform and on the order of the estimated coherence area. There as many as 71 islands in one bin at $75 \mathrm{~nm}^{2}$ that together make up $0.7 \%$ of the film area at $d^{\text {dep }}=0.76 \mathrm{~nm}$. Assuming the islands are round [see Figs. 3(a) and 3(b)], their diameters are $\sim 12$ and $25 \mathrm{~nm}$ for $d^{\text {dep }}=0.76$ and $0.89 \mathrm{~nm}$, respectively. As $d^{\text {dep }}$ nears $d_{\mathrm{IST}}$, individual islands coalesce to form larger islands. At the IST, $d^{\text {dep }}=1.07 \mathrm{~nm}$, a single $\mathrm{SC}$ island occupies $\simeq 50 \%$ of the film area. This island grows rapidly when $d^{\text {dep }} \simeq d_{\mathrm{IST}}$ as shown in Fig. 4(b). Similar results are obtained when this analysis is repeated for all the substrates studied. The dominant island film coverage increases sharply near $d_{\text {IST }}$ with very small changes in deposition: $10 \%-50 \%$ within $\Delta d^{\text {dep }}=0.04-0.14 \mathrm{~nm}$. Denoting $d_{50 \%}$ as the thickness at which the dominant island covers $50 \%$ of the film area, we show that $d_{50 \%} \simeq d_{\text {IST }}$ for experiments on all substrates studied [see Fig. 4(c)]. Thus, the coalescence of regions that are thick enough to support SC, as defined by a reference film, is necessary for the onset of SC in the NHC films.

This work indicates that thickness variations are primarily responsible for producing the CPI phase in $a$-Bi NHC films. Recent experiments on TiN films have shown that introducing a square array of holes can independently promote $\mathrm{CP}$ localization. ${ }^{30}$ Whether the accompanying holes in NHC films are playing a role in localization requires further investigation, but is at most a secondary effect.

The picture of insulating NHC films as composed of weakly coupled islands is qualitatively similar to the picture of the so-called granular film systems. Granular films, ${ }^{22,32}$ formed by the deposition of elemental vapor directly onto cryogenically 

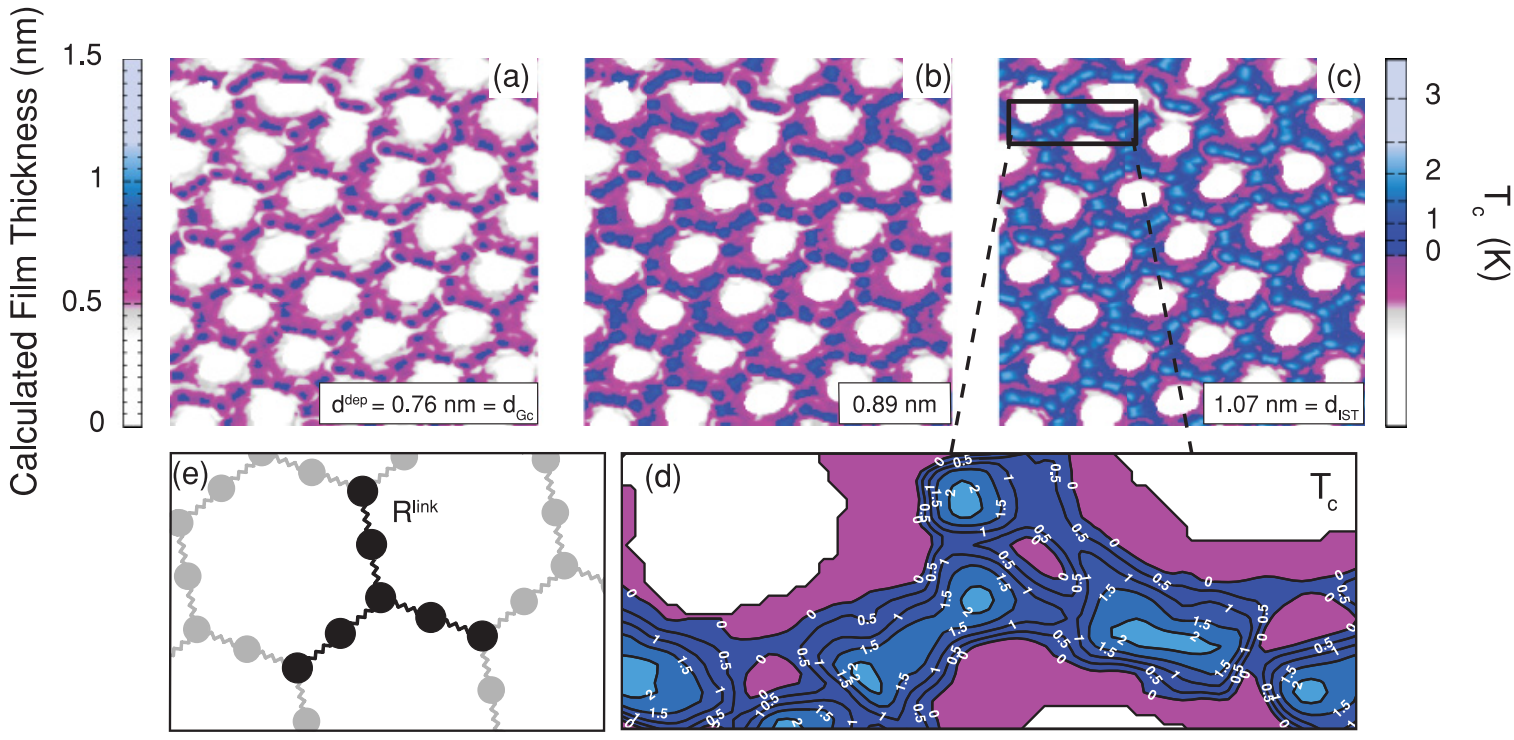

FIG. 3. (Color) Film thickness variations in NHC films. Calculated thickness variations in films deposited on the NHC substrate of Figs. 1(a) and 2(a). Film thickness coloring represents conductivity on a reference film: White is nonconductive $\left(d<d_{\mathrm{Gc}}^{\text {ref }}\right)$, pink is conductive $\left(d>d_{\mathrm{Gc}}^{\text {ref }}\right)$, and blue is superconductive $\left(d>d_{\mathrm{IST}}^{\text {ref }}\right)$. (a)-(c) Film thickness variation plots (a) at the onset of conduction, (b) for a representative insulating film [bold in Fig. 1(a)], and (c) at the IST. The critical temperatures for SC in reference films $\left(T_{\mathrm{c}}\right)$ corresponding to these film thicknesses are shown by the color bar on the right-hand side. $T_{\mathrm{c}}=0$ marks the IST and the maximum $T_{\mathrm{c}}$ seen in each of the panels is (a) $0.4 \mathrm{~K}$, (b) $1.3 \mathrm{~K}$, and (c) $2.2 \mathrm{~K}$. (d) $T_{\mathrm{c}}$ (in K) contours shown for a section of the film at $d^{\text {dep }}=d_{\mathrm{IST}}$. (e) Illustration of a wire array of resistors $R^{\text {link }}$ connecting SC islands. The basic element of the array is shown in black.
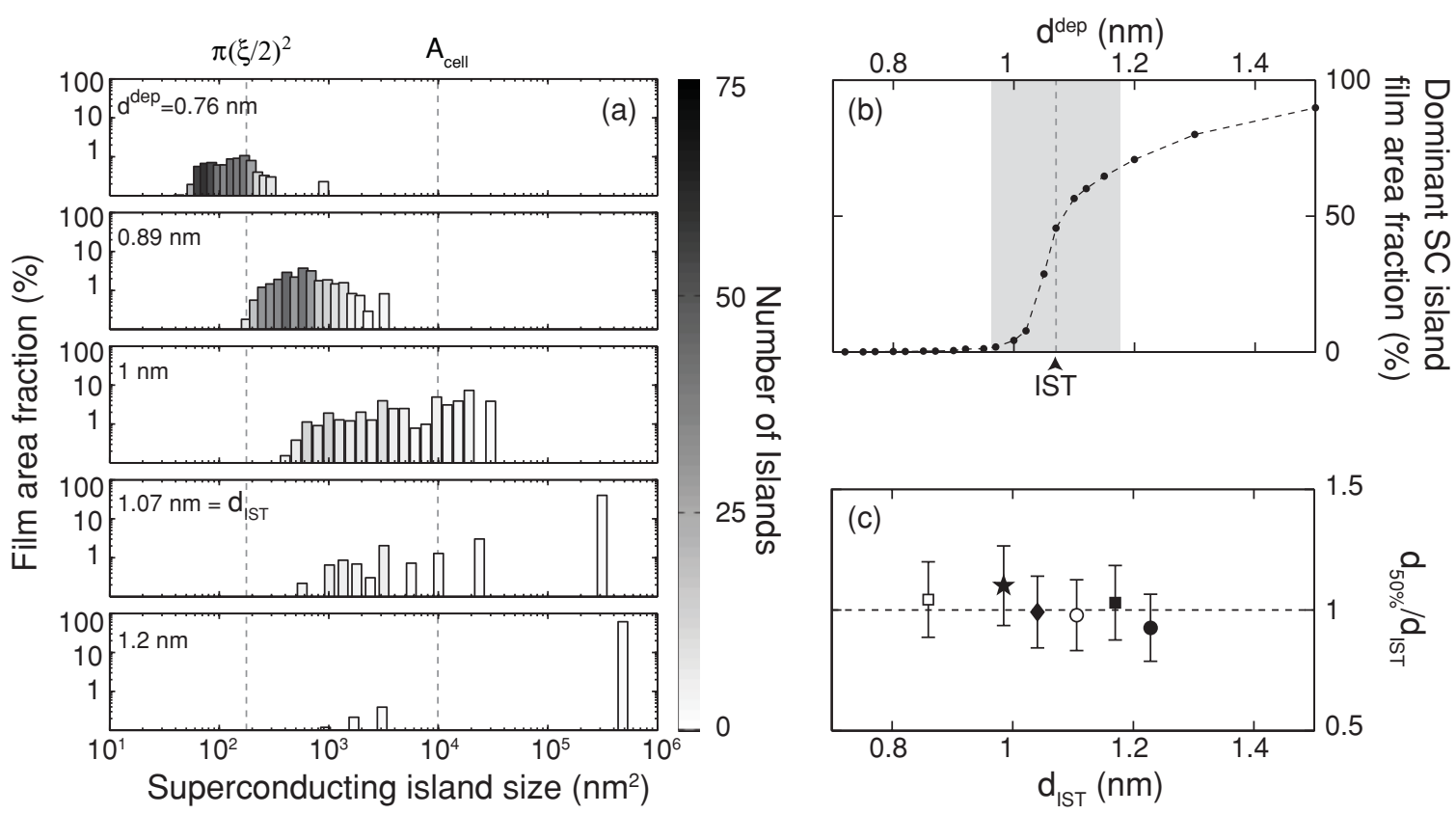

FIG. 4. Coalescence of superconducting islands. (a) SC island distributions showing individual island size, the number of islands of that size, and the fraction of the film area occupied by islands of that size for the substrate of Figs. 1(a), 2(a)-2(e), and 3. SC film regions are identified for $d(x, y)>d_{\mathrm{IST}}^{\text {ref }}=0.7 \mathrm{~nm}$ on a $1 \times 1 \mu \mathrm{m}$ image. Five different $d^{\text {dep }}$ are shown, three of which match those in Figs. 2 and $3 . d^{\text {dep }}=$ $1.07 \mathrm{~nm}$ is the IST. Vertical dashed lines are plotted to indicate a coherence area $\pi(\xi / 2)^{2}$ with $\xi=15 \mathrm{~nm}$, and the hole-array unit-cell area $A_{\text {cell }}=9900 \mathrm{~nm}^{2}$. (b) Film area coverage of the largest SC island for each deposition in (a) vs $d^{\text {dep }}$. The IST is shown as a vertical dashed line at $d^{\text {dep }}=1.07 \mathrm{~nm}$. The shaded region indicates a $10 \%$ uncertainty in the thickness measurement. (c) The analysis of (a) and (b) is repeated for films on all six different NHC substrates and here we plot the deposition thickness at which $50 \%$ of the film area is covered by a single SC island $d_{50 \%}$, compared to the experimental value $d_{\mathrm{IST}}$ for each substrate. The open circle plots the data in (a) and (b). 
cooled inert substrates, like glass, consist of weakly coupled crystalline grains. ${ }^{34}$ The granular film insulator also consists of localized Cooper pairs as shown by quasiparticle tunneling experiments. ${ }^{33}$ Nevertheless, there are strong differences between NHC and granular film transport that indicate two distinct states of localized Cooper pairs. Granular film transport appears to be dominated by intergrain quasiparticle tunneling. ${ }^{33}$ The temperature dependence of the sheet resistance rises exponentially and fits to a superconductorinsulator-superconductor quasiparticle tunneling model. The characteristic energy scale of this exponential rise is the superconducting energy gap, which does not change on approaching the IST. By contrast, transport in NHC films appears dominated by $\mathrm{CP}$ tunneling. The characteristic energy scale, the activation energy, is consistently smaller than the estimated SC energy gap in the films and steadily decreases on approaching the IST [see Fig. 1(d)]. Finally, granular films exhibit a negative magnetoresistance at low fields, which can be attributed to the field induced reduction of the superconducting energy gap. ${ }^{31}$ NHC films instead exhibit a positive magnetoresistance. ${ }^{19}$
Thus, the islanding in NHC films produces an insulator phase that differs from that in quench condensed granular films.

We have shown that the CPI phase in NHC $a$-Bi films results from variations in the film thickness over regions larger than $\xi$. Calculation of these thickness variations using the underlying substrate topography has allowed us to produce maps of superconducting coupling constant inhomogeneities in these films. Our observations support conjectures ${ }^{35-38}$ that $\mathrm{CP}$ islanding gives rise to the curious transport properties of the CPI phase. We anticipate that the detailed picture of the superconducting inhomogeneities presented here will aid microscopic calculations of these and other characteristics, such as the tunneling density of states, of the CPI state.

We are thankful to acknowledge helpful conversations with D. Feldman, N. Trivedi, T. Baturina, A. Goldman, and I. Beloborodov. This work was supported by the NSF through Grants No. DMR-0605797 and No. DMR-0907357, by the AFRL, the ONR, the AFOSR, and the WCU program at SNU, Korea.
*Low temperature lab, Aalto University, Espoo, Finland.

$\dagger$ Joint Quantum Institute, University of Maryland \& NIST, Gaithersburg, MD 20899, USA.

${ }^{\ddagger}$ Department of Electrical, Computer, and Energy Engineering, University of Colorado Boulder, Boulder, Colorado 80309, USA.

${ }^{1}$ V. F. Gantmakher and V. T. Dolgopolov, Phys. Usp. 53, 1 (2010).

${ }^{2}$ A. M. Finkelshtein, JETP Lett. 45, 46 (1987).

${ }^{3}$ D. Belitz, Phys. Rev. B 40, 111 (1989).

${ }^{4}$ D. B. Haviland, Y. Liu, and A. M. Goldman, Phys. Rev. Lett. 62, 2180 (1989).

${ }^{5}$ J. M. Valles Jr., S. Y. Hsu, R. C. Dynes, and J. P. Garno, Physica B 197, 522 (1994).

${ }^{6}$ J. A. Chervenak and J. M. Valles Jr., Phys. Rev. B 59, 11209 (1999).

${ }^{7}$ G. Sambandamurthy, L. W. Engel, A. Johansson, and D. Shahar, Phys. Rev. Lett. 92, 107005 (2004).

${ }^{8}$ T. I. Baturina, A. Y. Mironov, V. M. Vinokur, M. R. Baklanov, and C. Strunk, Phys. Rev. Lett. 99, 257003 (2007).

${ }^{9}$ A. F. Hebard and M. A. Paalanen, Phys. Rev. Lett. 65, 927 (1990).

${ }^{10}$ V. L. Pokrovsky, G. M. Falco, and T. Nattermann, Phys. Rev. Lett. 105, 267001 (2010).

${ }^{11}$ V. M. Vinokur, T. I. Baturina, M. V. Fistul, A. Y. Mironov, M. R. Baklanov, and C. Strunk, Nature (London) 452, 613 (2008).

${ }^{12}$ Y. Dubi, Y. Meir, and Y. Avishai, Phys. Rev. B 73, 054509 (2006).

${ }^{13}$ V. F. Gantmakher, M. V. Golubkov, V. T. Dolgopolov, G. E. Tsydynzhapov, and A. A. Shashkin, JETP Lett. 68, 363 (1998).

${ }^{14}$ B. Sacépé, C. Chapelier, T. I. Baturina, V. M. Vinokur, M. R. Baklanov, and M. Sanquer, Phys. Rev. Lett. 101, 157006 (2008).

${ }^{15}$ B. Sacépé, T. Dubouchet, C. Chapelier, M. Sanquer, M. Ovadia, D. Shahar, M. Feigel'man, and L. Ioffe, Nat. Phys. 7, 239 (2011).

${ }^{16}$ D. Kowal and Z. Ovadyahu, Physica C 468, 322 (2008).

${ }^{17}$ D. Kalok, A. Bilusic, T. I. Baturina, V. M. Vinokur, and C. Strunk, e-print arXiv:1004.5153v2.

${ }^{18}$ M. D. Stewart Jr., A. Yin, J. M. Xu, and J. M. Valles Jr., Science 318, 1273 (2007).

${ }^{19}$ H. Q. Nguyen, S. M. Hollen, M. D. Stewart, J. Shainline, A. Yin, J. M. Xu, and J. M. Valles Jr., Phys. Rev. Lett. 103, 157001 (2009).
${ }^{20}$ A. J. Yin, J. Li, W. Jian, A. J. Bennett, and J. M. Xu, Appl. Phys. Lett. 79, 1039 (2001).

${ }^{21}$ K. L. Ekinci and J. M. Valles, Phys. Rev. B 58, 7347 (1998).

${ }^{22}$ R. C. Dynes, J. P. Garno, and J. M. Rowell, Phys. Rev. Lett. 40, 479 (1978).

${ }^{23}$ M. D. Stewart Jr., A. Yin, J. M. Xu, and J. M. Valles Jr., Phys. Rev. B 77, 140501(R) (2008).

${ }^{24}$ M. D. Stewart Jr., H. Q. Nguyen, S. M. Hollen, A. Yin, J. M. Xu, and J. M. Valles Jr., Physica C 469, 774 (2009).

${ }^{25}$ M. D. Stewart Jr., Z. Long, J. M. Valles Jr., A. Yin, and J. M. Xu, Phys. Rev. B 73, 092509 (2006).

${ }^{26}$ S. J. Lee and J. B. Ketterson, Phys. Rev. Lett. 64, 3078 (1990).

${ }^{27}$ J. A. Chervenak, Ph.D. thesis, Brown University, Providence, RI, 1998.

${ }^{28}$ R. Fazio and H. van der Zant, Phys. Rep. 355, 235 (2001).

${ }^{29}$ Y. Takahide, R. Yagi, A. Kanda, Y. Ootuka, and S. I. Kobayashi, Phys. Rev. Lett. 85, 1974 (2000).

${ }^{30}$ T. I. Baturina, V. M. Vinokur, A. Y. Mironov, N. M. Chtchelkatchev, D. A. Nasimov, and A. V. Latyshev, Europhys. Lett. 93, 47002 (2011).

${ }^{31}$ I. S. Beloborodov, A. V. Lopatin, V. M. Vinokur, and K. B. Efetov, Rev. Mod. Phys. 79, 469 (2007).

${ }^{32}$ H. M. Jaeger, D. B. Haviland, B. G. Orr, and A. M. Goldman, Phys. Rev. B 40, 182 (1989).

${ }^{33}$ R. P. Barber, L. M. Merchant, A. LaPorta, and R. C. Dynes, Phys. Rev. B 49, 3409 (1994).

${ }^{34}$ K. L. Ekinci and J. M. Valles Jr., Phys. Rev. Lett. 82, 1518 (1999).

${ }^{35}$ A. Ghosal, M. Randeria, and N. Trivedi, Phys. Rev. Lett. 81, 3940 (1998).

${ }^{36}$ V. M. Galitski, G. Refael, M. P. A. Fisher, and T. Senthil, Phys. Rev. Lett. 95, 077002 (2005).

${ }^{37}$ Y. Dubi, Y. Meir, and Y. Avishai, Nature (London) 449, 876 (2007).

${ }^{38}$ M. V. Feigel'man, L. B. Ioffe, V. E. Kravtsov, and E. A. Yuzbashyan, Phys. Rev. Lett. 98, 027001 (2007). 